\title{
CONVERGENT SUBSEQUENCES FROM SEQUENCES OF FUNCTIONS(1)
}

BY

\section{JAMES L. THORNBURG}

ABSTRACT. Let $\left\{y_{k}\right\}$ be a sequence of functions, $y_{k} \in \mathbf{\Pi}_{s} \in S E_{s}$ where $S$ is a nonempty subset of the $l$-dimensional Euclidean space and $E_{s}$ is an ordered vector space with positive cone $K_{s}$. If $y_{k} \in \mathbf{\Pi}_{s \in S} E_{s}$, sufficient conditions are given that $\left\{y_{k}\right\}$ have a subsequence $\left\{h_{k}\right\}$ such that for each $t \in S$ the sequence $\left\{h_{k}(t)\right\}$ is monotone for $k$ sufficiently large, depending on $t$. If each $E_{s}$ is an ordered topological vector space, sufficient conditions are given that $\left\{y_{k}\right\}$ has a subsequence $\left\{h_{k}\right\}$ such that for every $t \in S$ the sequence $\left\{h_{k}(t)\right\}$ is either monotone for $k$ sufficiently large depending on $t$, or else the sequence $\left\{h_{k}(t)\right\}$ is convergent. If $E_{s}=B$ for each $s$ and $B$ a Banach space then a definition of bounded variation is given so that if $\left\{y_{k}\right\}$ is uniformly norm bounded and the variation of the functions $y_{k}$ is uniformly bounded then there is a convergent subsequence $\left\{h_{k}\right\}$ of $\left\{y_{k}\right\}$. In the case $E_{s}=E$ for each $s \in S$ and $E$ is such that bounded monotone sequences converge then the given conditions imply the existence of a subsequence $\left\{h_{k}\right\}$ of $\left\{y_{k}\right\}$ which converges for each $t \in S$.

1. Introduction. The terminology used in this paper relating to ordered vector spaces will agree with that of Peressini [10] unless stated otherwise or explicitly defined. In particular, the definitions of ordered vector space $E$, positive cone $K$ of $E$, ordered interval $[a, b]$ in $E$ where $a \leq b$, ordered bounded subset of $E$, majorized subset of $E$, ordered topological vector space $E$, ordered locally convex space $E$, and normal positive cone $K$ of a topological vector space $E$ agree with [10]. An ordered Banach space $B$ will be a Banach space which is also an ordered vector space and thus is an ordered topological vector space. We note that we do not require the positive cone $K$ of a topological vector space $E$ to be closed as is done, for example, in

Received by the editors April 19, 1974.

AMS (MOS) subject classifications (1970). Primary 40A05, 26A45; Secondary $26 A 46$.

Key words and phrases. Sequences of functions, convergence of subsequences, monotonicity of subsequences, variation, functions of bounded variation, ordered topological vector spaces.

(1) This work is in partial fulfillment of the requirements for the Doctor of Philosophy degree at the University of Missouri, Columbia. 
Shaefer [16]. The $l$-dimensional Euclidean space $R^{l}$ will be considered with the usual norm unless otherwise stated, the open sphere will be $D(t, \delta)=$ $\left\{x \in R^{l}:\|t-x\|<\delta\right\}$ with center $t$ and radius $\delta$ and the order will be determined by some positive cone

$$
\begin{array}{r}
Q_{k}=\left\{\left(x^{(1)}, x^{(2)}, \ldots, x^{(l)}\right): x^{(l)} \geq 0, x^{(i)} \geq 0 \text { for } t_{2}=0, x^{(i)} \leq 0\right. \text { for } \\
\left.t_{i}=1 \text { where } k=1+t_{1}+2 t_{2}+2^{2} t_{3}+\cdots+2^{(l-2)} t_{l-1}\right\}, \\
1 \leq k \leq 2^{l-1} .
\end{array}
$$

The interior of the positive cone will be

$$
\begin{aligned}
Q_{k}^{\circ}=\left\{\left(x^{(1)}, x^{(2)}, \ldots, x^{(l)}\right):\right. & x^{(l)}>0, x^{(i)}>0 \text { if } t_{i}=0, x^{(i)}<0 \text { if } t_{i}=1 \\
& \text { for } \left.k=1+t_{1}+2 t_{2}+2^{2} t_{3}+\cdots+2^{(l-2)} t_{l-1}\right\} .
\end{aligned}
$$

The translate of any $l-1$ dimensional subspace will be called a hyperplane in $R^{l}$ and if it is of the form $\left\{\left(\alpha^{(1)}, a^{(2)}, \ldots, \alpha^{(l)}\right): \alpha^{(i)}=c\right.$ for $i$ fixed and some constant $c\}$ it will be called a regular hyperplane. A closed interval will be

$$
I=[\alpha, \beta]=\left\{\left(x^{(1)}, x^{(2)}, \ldots, x^{(l)}\right): \alpha^{(i)} \leq x^{(i)} \leq \beta^{(i)}, i=1,2, \ldots, l\right\}
$$

where $a=\left(\alpha^{(1)}, \alpha^{(2)}, \ldots, \alpha^{(l)}\right), \beta=\left(\beta^{(1)}, \beta^{(2)}, \ldots, \beta^{(l)}\right)$ and $\alpha^{(i)}<\beta^{(i)}$ for $i=1,2, \ldots, l$. The vertices of $I$ will be $\left\{x_{1}, x_{2}, \ldots, x_{2} l\right.$ where

$$
x_{k}=\left(\gamma^{(1)}, \gamma^{(2)}, \ldots, \gamma^{(l)}\right), \quad \gamma^{(i)}=\left\{\begin{array}{l}
\alpha^{(i)} \text { if } t_{i}=0, \\
\beta^{(i)} \text { if } t_{i}=1,
\end{array} \quad i=1,2, \ldots, l,\right.
$$

and

$$
k=1+t_{1}+2 t_{2}+\cdots+2^{(l-1)} t_{l}
$$

The set $\left\{\left(\gamma^{(1)}, \gamma^{(2)}, \ldots, \gamma^{(l)}\right): \gamma^{(m)}=\alpha^{(m)}\right.$ for $m$ fixed and $\alpha^{(i)} \leq \gamma^{(i)} \leq \beta^{(i)}$ for $i \neq m\}$ will be called a minimum bounding edge of $I$ and $\left\{\left(\gamma^{(1)}, \gamma^{(2)}, \ldots\right.\right.$, $\left.\gamma^{(l)}\right): \gamma^{(m)}=\beta^{(m)}$ for $m$ fixed and $\alpha^{(i)} \leq \gamma^{(i)} \leq \beta^{(i)}$ for $\left.i \neq m\right\}$ will be called a maximum bounding edge of $l$.

Two elements $x_{k}$ and $x_{j}$ in an ordered vector space $E$ with positive cone $K$ will be comparable if $x_{k}-x_{j} \in K$ or $x_{j}-x_{k} \in K$ holds. By a monotone nondecreasing sequence $\left\{x_{k}\right\}$ in an ordered vector space $E$ we mean $x_{k+1}-x_{k} \in K$ holds for all $k$. A monotone nonincreasing sequence is similarly defined and a sequence is called monotone if it is either monotone nondecreasing ot monotone nonincreasing. 
A sequence $\left\{x_{k}\right\}$ in an ordered vector space $E$ will be said to be eventually monotone nondecreasing if there exists an integer $k_{0}$ so that $x_{k+1}$ $x_{k} \in K$ holds for all $k \geq k_{0}$. An eventually monotone nondecreasing sequence is similarly defined and a sequence is called eventually monotone if it is either eventually monotone nondecreasing or eventually monotone nonincreasing.

If $\left\{y_{k}\right\}$ is a sequence of functions, $y_{k} \in \Pi_{s \in S} E_{s}$ where $S$ is a nonempty subset of $R^{l}$ and $E_{s}$ is an ordered vector space with positive cone $K_{s}$ then we say that the sequence $\left\{y_{k}\right\}$ is a monotone nondecreasing sequence of functions on $S$ if the sequence $\left\{y_{k}(s)\right\}$ is a monotone nondecreasing sequence in $E_{s}$ for each $s \in S$. A monotone nonincreasing sequence of functions on $S$ is similarly defined and a sequence of functions is said to be monotone on $S$ if it is either monotone nondecreasing or monotone nonincreasing.

If $\left\{y_{k}\right\}$ is a sequence of functions, $y_{k} \in \Pi_{s \in S} E_{s}$, where $S$ is a nonempty subset of $R^{l}$ and $E_{s}$ is an ordered vector space with positive cone $K_{s}$ then we say that the sequence of functions $\left\{y_{k}\right\}$ is an eventually monotone nondecreasing sequence on $S$ if $\left\{y_{k}(s)\right\}$ is eventually monotone for each $s \in S$.

Let $\left\{y_{k}\right\}$ be a sequence of functions, $y_{k} \in \Pi_{s \in S} E_{s}$ where $S$ is a nonempty subset of $R^{l}$ and each $E_{s}$ is an ordered vector space. Sufficient conditions that $\left\{y_{k}\right\}$ have a subsequence $\left\{h_{k}\right\}$ such that for each $s \in S,\left\{h_{k}(s)\right\}$ is eventually monotone in $E_{s}$ are given in Theorem 4.1. In case each $E_{s}$ is a sequentially complete ordered locally convex space, Theorem 4.4 gives sufficient conditions that there be a subsequence $\left\{h_{k}\right\}$ of $\left\{y_{k}\right\}$ such that for every $s \in S$ the sequence $\left\{h_{k}(s)\right\}$ is either eventually monotone or else convergent in $E_{s}$. Additional hypotheses are given in Theorem 5.1 to insure the existence of a subsequence $\left\{h_{k}\right\}$ of $\left\{y_{k}\right\}$ such that $\left\{h_{k}(s)\right\}$ converges in $E_{s}$ for each $s \in S$. This generalizes the results in [15] and gives, in Theorem 5.4, a necessary and sufficient condition that a sequence of functions from a subset of $l$-dimensional Euclidean space into $q$-dimensional Euclidean space have a subsequence that is pointwise convergent.

For $\left\{y_{k}\right\}$ a sequence of functions, $y_{k}: S \rightarrow B$ where $S$ is a nonempty subset of $R^{l}$ and $B$ is an ordered Banach space, a definition of variation is given in $\S 3$ so that Theorem 5.2 yields a generalized Helly selection theorem as a corollary.

2. Preliminary results. We will refer to a result of Ramsey [11, Theorem A, p. 264] or [12, Theorem A, p. 82] repeatedly so we will include its statement here.

Theorem 2.1. Let $\Gamma$ be an infinite class, $u$ and $r$ positive integers, and let those subclasses of $\Gamma$ which have exactly $r$ members, or, as we may 
say, let all r-combinations of the members of $\Gamma$ be divided in any manner into $u$ mutually exclusive classes $\epsilon_{i}(i=1,2, \ldots, u)$, so that every r-combination is a member of one and only one $C_{i}$. Then, assuming the axiom of selections, $\Gamma$ must contain an infinite subclass $\Delta$ such that all the r-combinations of the members of $\Delta$ belong to the same $C_{i}$.

Corollary 2.2. Let $J$ be a nonempty subset of $R^{l}$ and $\left\{f_{k}\right\}$ be a sequence of functions, $f_{k} \in \Pi_{t \in J} E_{t}$ where each $E_{t}$ is an ordered vector space with positive cone $K_{i}$. If $f_{k}(t)$ and $f_{j}(t)$ are comparable for each $t \in J$ and $k, j=$ $1,2, \ldots$, then either there is a subsequence $\left\{h_{j}\right\}$ of $\left\{f_{k}\right\}$ such that $\left\{h_{j}\right\}$ is a monotone subsequence on $J$ or else there is a subsequence $\left\{h_{j}\right\}$ of $\left\{f_{k}\right\}$ such that if $i \neq j$ there are $t, \tau \in J$ depending on $i, j$ with $h_{i}(t)-h_{j}(t) \in K_{t^{\prime}} h_{i}(t)$ $\neq h_{j}(t)$ and $h_{j}(\tau)-h_{i}(\tau) \in K_{\tau}, h_{j}(\tau) \neq h_{i}(\tau)$.

The proof of this corollary follows in a similar manner to that of Corollary 2.3 in [14].

Corollary 2.3. Let $J$ be a nonempty subset of $R^{l}$ and $\left\{f_{k}\right\}$ be a sequence of functions, $f_{k} \in \Pi_{t} \in J, E_{t}$, where each $E_{t}$ is an ordered topological vector space. If $W_{t}$ is a circled neighborhood of $\theta_{t}$ in $E_{t}$ then either there is a subsequence $\left\{h_{j}\right\}$ of $\left\{f_{k}\right\}$ such that for $i \neq j, h_{j}(t)-h_{i}(t) \in W_{t}$ for all $t \in J$ or else there is a subsequence $\left\{h_{j}\right\}$ of $\left\{f_{k}\right\}$ such that for $i \neq j, h_{j}(t)-h_{i}(t) \notin w_{t}$ for some $t \in J$ depending on $i, j$.

The proof of this corollary follows in a similar manner to that of Corollary $2 . \dot{3}$ in [14].

3. Variation. In [14] where sequences of functions from the real numbers into the real numbers were considered, a result yielding the Helly selection theorem as a corollary was given. Then in [15] where the functions were from the real numbers into ordered Banach spaces, a generalized Helly selection theorem was given as a corollary. Browne [1] considers functions from l-dimensional Euclidean space into the real numbers and with one definition of variation gets another generalized Helly selection theorem. Four definitions of variation are given here and their relationship considered when the functions are from $l$-dimensional Euclidean space into an ordered Banach space.

Consider the interval $I=[a, \beta] \subset R^{l}$ and $a, b \in I$ with $a^{(i)}<b^{(i)}$ for $i=1,2, \ldots, l$, then $J=[a, b]$ will be a subinterval of $I$. A subdivision of $[\alpha, \beta]$ will consist of a finite number of subintervals $J_{1}, J_{2}, \ldots, J_{m}$ of $I$ such that $\bigcup_{i=1}^{m} J_{i}=I$ and for $k \neq j, J_{k} \cap J_{j}$ is either empty, a point, or is a $p$-dimensional interval where $1 \leq p \leq l-1$. A refinement of the subdivision $\left\{J_{1}, J_{2}, \ldots, J_{m}\right\}$ will be a subdivision obtained by subdividing one or more 
of the $J_{i}$ 's into two or more subintervals. A subdivision $\left\{J_{1}, J_{2}, \ldots, J_{m}\right\}$ will be called regular if all of the minimum bounding edges of $J_{n}=\left[a_{n}, b_{n}\right]$,

$$
\left\{\left(\gamma^{(1)}, \gamma^{(2)}, \ldots, \gamma^{(l)}\right): \gamma^{(j)}=a_{n}^{(j)}, a^{(i)} \leq \gamma^{(i)} \leq b^{(i)} \text { for } i \neq j\right\}
$$

and the maximum bounding edges of $J_{n}$,

$$
\left\{\left(\gamma^{(1)}, \gamma^{(2)}, \ldots, \gamma^{(l)}\right): \gamma^{(j)}=b_{n}^{(j)}, a_{n}^{(i)} \leq \gamma^{(i)} \leq b^{(i)} \text { for } i \neq j\right\}
$$

for $n=1,2, \ldots, m$ are extended to

$$
\left\{\left(\gamma^{(1)}, \gamma^{(2)}, \ldots, \gamma^{(l)}\right): \gamma^{(j)}=a_{n}^{(j)}, \alpha^{(i)} \leq \gamma^{(i)} \leq \beta^{(i)} \text { for } i \neq j\right\}
$$

and

$$
\left\{\left(\gamma^{(1)}, \gamma^{(2)}, \ldots, \gamma^{(l)}\right): \gamma^{(j)}=b_{n}^{(j)}, a^{(i)} \leq \gamma^{(i)} \leq \beta^{(i)} \text { for } i \neq j\right\},
$$

respectively, and the subdivision is not refined. A set $X=\left\{x_{1}=\alpha, x_{2}, x_{3}\right.$, $\left.\ldots, x_{n-1}, x_{n}=\beta\right\} \subset I=[\alpha, \beta]$ will be called a partition of $I$. The subdivision whose bounding edges are formed by the intersection of $I$ with all the regular hyperplanes through $\left\{x_{1}, x_{2}, \ldots, x_{n}\right\}$ will be called the subdivision determined by the partition $\left\{x_{1}, x_{2}, \ldots, x_{n}\right\}$. The set of vertices of all the subintervals of a subdivision $\left\{J_{1}, J_{2}, \ldots, J_{m}\right\}$ will be the partition determined by the subdivision. A partition $X$ will be called regular if the set of vertices of the subintervals in the subdivision determined by $X$ is again $X$. A partition $X_{0}$ will be said to be regularized to $X$ if $X$ consists of the vertices of the subintervals in the subdivision determined by the partition $X_{0}$.

For a function $f: I \rightarrow B$ where $B$ is an ordered Banach space, $I=[\alpha, \beta]$ $C R^{l}, \alpha=\left(\alpha^{(1)}, \alpha^{(2)}, \ldots, \alpha^{(l)}\right), \beta=\left(\beta^{(1)}, \beta^{(2)}, \ldots, \beta^{(l)}\right)$ and $\alpha^{(i)}<\beta^{(i)}$ for $i=1,2, \ldots, l$ consider the following definitions of the variation of $f$ on I.

Definition 3.1. Let $X=\left\{x_{1}, x_{2}, \ldots, x_{n}\right\}$ be called an allowable partition of $I$ if $X$ is such that $x_{k+1}-x_{k} \in Q_{j}$ for $k=1,2, \ldots, n-1, Q_{j}$ is a fixed positive cone and $x_{1}$ is the smallest vertex of $I$ and $x_{n}$ is the largest vertex of $I$ as determined by $Q_{j}$. The variation of $f$ on $I$, denoted by $V_{1}(f ; I)$, is given by

$$
V_{1}(f ; l)=\sup _{x} \sum_{k=1}^{n-1}\left\|f\left(x_{k}\right)-f\left(x_{k+1}\right)\right\|
$$

where the supremum is over all allowable partitions $X$ ordered by a positive cone $Q_{j}$ for $j=1,2, \ldots, 2^{l-1}$. The function $f$ will be said to be of bounded variation, denoted $f \in B V_{1}$, if $V_{1}(f ; I)<+\infty$. 
An interval $l=[\alpha, \beta] \subset R^{l}$ with the operation $*$ given by

$$
x * y=\left(\max \left\{x^{(1)}, y^{(1)}\right\}, \max \left\{x^{(2)}, y^{(2)}\right\}, \ldots, \max \left\{x^{(l)}, y^{(l)}\right\}\right)
$$

forms an idempotent semigroup with identity $a$. For $x \in I$, the conventions $x^{1}=x$ and $x^{0}=\alpha$ will be assumed. Thus a special case of the definition of variation given in [9] is given in the following:

Definition 3.2 (Newman). Let $T_{n}$ denote the Boolean algebra of all $n$ tuples of zeros and ones. Let $\sigma, \tau \in T_{n}$ be such that $\tau \geq \sigma$ if $\tau(i) \geq \sigma(i)$ for $i=1,2, \ldots, n$ and

$$
\mu(\sigma, \tau)= \begin{cases}(-1)^{|\tau|-|\sigma|,} & \tau \geq \sigma, \\ 0, & \text { otherwise }\end{cases}
$$

where $|\sigma|$ denotes the number of ones in the $n$-tuple $\sigma$. Then for $X=\left\{x_{1}, x_{2}\right.$, $\left.\ldots, x_{n}\right\}$ any partition of $I$ with $x_{1}=\alpha$ and $x_{n}=\beta$ define

$$
L(X, \sigma) f=\sum_{\tau \in T_{n}} \mu(\sigma, \tau) f\left(\prod_{i=1}^{n} x_{i}^{\tau(i)}\right)
$$

where $\Pi$ is the product under the operation $*$ making $I$ an idempotent semigroup. Then the variation of $f$ on $I$, denoted by $V_{2}(f ; I)$, is given by

$$
V_{2}(f ; I)=\sup _{X} \sum_{\sigma \in T_{n}}\|L(X, \sigma) f\|
$$

where the supremum is taken over all finite partitions $X$ of $I$. The function $f$ will be said to be of bounded variation, denoted $f \in B V_{2}$, if $V_{2}(f ; I)<+\infty$.

Definition 3.3 (Arzela). Let $X=\left\{x_{1}, x_{2}, \ldots, x_{n}\right\}$ be called a permissible partition of $I$ if $X$ is such that $x_{1}=\alpha, x_{n}=\beta$ and $x_{k+1}-x_{k} \in Q_{1}$ for $k=$ $1,2, \ldots, n-1$. Then the variation of $f$ on $I$, denoted by $V_{3}(f ; I)$, is given by

$$
V_{3}(f ; l)=\sup _{X} \sum_{x=1}^{n-1}\left\|f\left(x_{k}\right)-f\left(x_{k+1}\right)\right\|
$$

where the supremum is taken over all permissible partitions $X$ of $I$. The function $f$ will be said to be of bounded variation, denoted $f \in B V_{3}$, if $V_{3}(f ; I)<+\infty$.

Definition 3.4 (Lebesgue). Let $J=[a, b]$ be a subinterval of $I$ and

$$
\begin{aligned}
\Delta_{r} f\left(x^{(1)},\right. & \left.x^{(2)}, \ldots, x^{(r-1)}, x^{(r+1)}, \ldots, x^{(l)}\right) \\
= & f\left(x^{(1)}, \ldots, x^{(r-1)}, b^{(r)}, x^{(r+1)}, \ldots, x^{(l)}\right) \\
& -f\left(x^{(1)}, \ldots, x^{(r-1)}, a^{(r)}, x^{(r+1)}, \ldots, x^{(l)}\right)
\end{aligned}
$$


for $r=1,2, \ldots, l$ and $\Delta_{f} J=\Delta_{1} \Delta_{2} \ldots \Delta_{l} f$. The variation of $f$ on $I$, denoted $V_{4}(f ; l)$, is given by

$$
V_{4}(f ; I)=\sup _{Z} \sum_{J \in Z}\left\|\Delta_{f} J\right\|
$$

where the supremum is taken over all subdivisions $Z$ of $I$. The function $f$ will be said to be of bounded variation, denoted $f \in B V_{4}$, if $V_{4}(f ; l)<+\infty$.

If $X=\left\{x_{1}, x_{2}, \ldots, x_{n}\right\}$ is a permissible partition for the calculation of $V_{3}(f ; l)$ it is also an allowable partition for $V_{1}(f ; I)$ so the calculation of $V_{1}(f ; I)$ is the supremum over a larger set than is the calculation of $V_{3}(f ; I)$. Thus $f \in B V_{1}$ implies that $f \in B V_{3}$. The converse of this statement does not hold however. Consider the function $f: I \rightarrow R, I=[0,1] \subset R^{2}$ defined by

$$
f(x, y)= \begin{cases}\sin (1 / x) & \text { if } x+y=1, \\ 0, & \text { otherwise. }\end{cases}
$$

Since $\sin (1 / x)$ is not of bounded variation on $[0,1] \subset R, f(x, y)$ is not of bounded variation by Definition 1 . However any partition ordered by $Q_{1}$ contains at most one point where $f(x, y)$ is nonzero so that $V_{3}(f ; I) \leq 2$.

To determine the relationship between $V_{2}(f ; I)$ and $V_{4}(f ; I)$ some preliminary results are necessary. In the calculation of $V_{2}(f ; l)$ the elements of $T_{1}$ will be denoted $\sigma_{1}^{(1)}=(1)$ and $\sigma_{1}^{(2)}=(0)$. Assume that the elements of $T_{n-1}$ have been numbered, then those of $T_{n}$ will be given by $\sigma_{n}^{(1)}=\left(\sigma_{n-1}^{(1)}, 1\right)$, $\sigma_{n}^{(2)}=\left(\sigma_{n-1}^{(1)}, 0\right), \ldots, \sigma_{n}^{(k)}=\left(\sigma_{n-1}^{(m)}, 1\right)$ for $k^{n}=2^{m}-1$ and $\sigma_{n}^{(k)}=\left(\sigma_{n-1}^{(m)}, 0\right)$ for $k=2 m$ where $k=1,2, \ldots, 2^{n}$. The $i$ th coordinate of $\sigma_{n}^{(k)}$ will be denoted $\sigma_{n}^{(k)}(i)$. The subscript $n$ will be deleted from $\sigma_{n}^{(k)}$ when it is clear that $\sigma_{n}^{(k)} \in T_{n}$.

Note 1. Let $f: I \rightarrow B, I=[a, \beta] \subset R^{l}$ and $X=\left\{x_{1}, x_{2}, \ldots, x_{2}\right\}$ be the vertices of $I$ with $x_{1}=\alpha, x_{2} l=\beta$ and $x_{k}=\left(\gamma^{(1)}, \gamma^{(2)}, \ldots, \gamma^{(l)}\right)$ where

$$
\gamma^{(i)}= \begin{cases}\alpha^{(i)} & \text { if } t_{i}=0 \\ \beta^{(i)} & \text { if } t_{i}=1\end{cases}
$$

for $i=1,2, \ldots, l$ and $k=1+t_{1}+2 t_{2}+\cdots+2^{(l-1)} t_{l}$. Then $L\left(X, \sigma^{2^{\left(2^{l}-1\right)}}\right) f$ $=\Delta_{f} I$ where $\Delta_{f} I$ is as in Definition 5.4.

Proof. Let

$$
\Gamma_{m}=\left\{\sigma^{(k)}: \sigma^{(k)} \in T_{2 l},\left(\prod_{i=1}^{2 l} x_{i}^{\sigma^{(k)(i)}}\right)=x_{m}\right\}
$$


for $m=1,2, \ldots, 2^{l}$, let $\sigma^{\left(p_{m}\right)} \in \Gamma_{m}$ be such that $\sigma^{(k)} \leq \sigma^{\left(p_{m}\right)}$ for all $\sigma^{(k)}$ $\epsilon \Gamma_{m}$, and let $\eta=\sigma^{2\left(2^{l}-1\right)}$. Since $\sigma^{(k)}(1)=0$ for $k \geq 2^{\left(2^{l}-1\right)}$ and $\eta(1)=1$, we have that $\mu\left(\eta, \sigma^{(k)}\right)=0$ for $k>2^{\left(2^{l}-1\right)}$.

$$
\begin{aligned}
L\left(X, \sigma^{\left.2^{(2 l}-1\right)}\right) f= & \sum_{\sigma \in T_{2} l} \mu\left(\sigma^{\left.2^{(2 l}-1\right)} \sigma\right) f\left(\prod_{i=1}^{2^{l}} x_{i}^{\sigma(i)}\right)=\sum_{\sigma \in \Gamma_{1}} \mu(\eta, \sigma) f\left(\prod_{i=1}^{2^{l}} x_{i}^{\sigma(i)}\right) \\
& +\sum_{\sigma \in \mathbf{\Gamma}_{2}} \mu(\eta, \sigma) f\left(\prod_{i=1}^{2^{l}} x_{i}^{\sigma(i)}\right)+\cdots+\sum_{\sigma \in \mathbf{\Gamma}_{2} l} \mu(\eta, \sigma) f\left(\prod_{i=1}^{2 l} x_{i}^{\sigma(i)}\right) .
\end{aligned}
$$

Hence it will suffice to show that

$$
\sum_{\sigma \in \Gamma_{m}} \mu(\eta, \sigma) f\left(\prod_{i=1}^{{ }^{l}} x_{i}^{\sigma(i)}\right)=(-1)^{d} f_{m}\left(x_{m}\right)
$$

where $d_{m}$ is the number of $t_{i}=1$ for $m=1+t_{1}+2 t_{2}+\cdots+2^{(l-1)} t_{l}$.

For $d_{m}=0,1,2, \ldots, l$ and $n=0,1, \ldots, 2^{l}-1$ let $N\left(d_{m}, n\right)$ be the number of distinct elements $\sigma^{(p)} \in \Gamma_{m}$ such that $\mu\left(\eta, \sigma^{(p)}\right) \neq 0$ and $\sigma^{(p)}(i)=$ 1 for $n$ values of $i \neq 1$. The number of $i \neq 1$ such that $\sigma^{\left(p_{m}\right)}(i)=1$ is $2^{d_{m}}$ - 1 and $\sum_{n=0}^{2 l} N\left(d_{m}, n\right)$ is the number of elements $\sigma \in \Gamma_{m}$ such that $\mu(\eta, \sigma)$ $\neq 0$ for $d_{m}=0,1,2, \ldots, l$. Thus $N(0,0)=1$ and

$$
\begin{aligned}
N\left(d_{m}, n\right)= & \left(\begin{array}{c}
2^{d_{m}}-1 \\
n
\end{array}\right)-\left(\begin{array}{c}
d_{m} \\
1
\end{array}\right) N\left(d_{m}-1, n\right)-\left(\begin{array}{c}
d_{m} \\
2
\end{array}\right) N\left(d_{m}-2, n\right) \\
& -\cdots-\left(\begin{array}{c}
d_{m} \\
d_{m}-1
\end{array}\right) N(1, n)-\left(\begin{array}{c}
d_{m} \\
d_{m}
\end{array}\right) N(0, n)
\end{aligned}
$$

for $n \leq 2^{d_{m}}-1$ and $N\left(d_{m}, n\right)=0$ for $n>2^{d_{m}}-1$. Now suppose $\sigma^{(k)} \geq \eta$ and contains $n+1$ ones so that $\mu\left(\eta, \sigma^{(k)}\right)=(-1)^{n}$. Hence the note will hold if

$$
\sum_{j=0}^{2 l-1} N\left(d_{m}, 2 j\right)-\sum_{j=1}^{2 l-1} N\left(d_{m}, 2 j-1\right)=(-1)^{d_{m}}
$$

for $d_{m}=0,1,2, \ldots, l$ since then (2) is true.

For $d_{m}=0$ it holds. Assume that (3) holds for $d_{m} \leq r-1$. Then for $d_{m}=r$, 


$$
\begin{aligned}
& \sum_{j=0}^{2(2 l-1)} N(r, 2 j)-\sum_{j=1}^{2 l-1} N(r, 2 j-1) \\
& \quad=\sum_{j=0}^{2 l-1}\left[\left(\begin{array}{c}
2^{r}-1 \\
2 j
\end{array}\right)-\left(\begin{array}{l}
r \\
1
\end{array}\right) N(r-1,2 j)-\left(\begin{array}{l}
r \\
2
\end{array}\right) N(r-2,2 j)-\cdots-\left(\begin{array}{l}
r \\
r
\end{array}\right) N(0,2 j)\right] \\
& \quad-\sum_{j=1}^{2 l-1}\left[\left(\begin{array}{c}
2^{r}-1 \\
2 j-1
\end{array}\right)-\left(\begin{array}{l}
r \\
1
\end{array}\right) N(r-1,2 j-1)-\left(\begin{array}{l}
r \\
2
\end{array}\right) N(r-2,2 j-1)\right. \\
& =-\left(\begin{array}{l}
r \\
1
\end{array}\right)(-1)^{r-1}-\left(\begin{array}{l}
r \\
2
\end{array}\right)(-1)^{r-2} \cdots-\left(\begin{array}{c}
r \\
r-1
\end{array}\right)(-1)^{1}-1
\end{aligned}
$$

where we define

$$
\left(\begin{array}{c}
2^{r}-1 \\
b
\end{array}\right)=0 \text { if } b>2^{r}-1
$$

Thus we have that

$$
-r(-1)^{r-1}-\cdots:\left(\begin{array}{c}
r \\
r-1
\end{array}\right)(-1)^{1}-1=-[(-1)+1]^{r}+(-1)^{r}+1-1=(-1)^{r}
$$

so the desired result holds.

Note 2. Let $f: I \rightarrow B, I=[\alpha, \beta] \subset R^{l}$ and let $X=\left\{x_{1}, x_{2}, \ldots, x_{2} l\right\}$ be the vertices of $I$ numbered as in Note 1. For $\Gamma_{m}$ as in (1), $\sigma^{\left(p_{m}\right)} \in \Gamma_{m} ; \sigma^{\left(p_{m}\right)}$ $\geq \sigma^{(k)}$ for all $\sigma^{(k)} \in \Gamma_{m}$ we have that $L\left(X, \sigma^{\left(p_{m}\right)}\right) f=\Delta_{f} I_{m}$ where $I_{m}$ is the interval whose vertices are $\left\{x_{i}: x_{i}-x_{m} \in Q_{1}\right\}$.

Proof. By definition

$$
L\left(X, \sigma^{\left.p_{m}\right)}\right)=\sum_{\sigma^{(k)} \in T_{2} l} \mu\left(\sigma^{\left(p_{m}\right)}, \sigma^{(k)}\right) f\left(\prod_{i=1}^{2 l} x_{i}^{\sigma^{(k)}(i)}\right)
$$

We need only consider those $\sigma^{(k)} \geq \sigma^{\left(p_{m}\right)}$ since $\mu\left(\sigma^{\left(p_{m}\right)}, \sigma^{(k)}\right)=0$ otherwise. The set $\left\{x_{r}: \Pi_{i=1}^{2^{l}} x_{i}^{\sigma(k)(i)}=x_{r}\right\}$ for some $\sigma^{(k)} \geq \sigma^{\left(p_{m}\right)}=\left\{x_{r}: x_{r}-x_{m} \in Q_{1}\right\}$. Now let

$$
\Delta_{r}=\left\{\sigma^{(k)}: \prod_{i=1}^{2 l} x_{i}^{\sigma^{(k)}(i)}=x_{r}, \quad \sigma^{(k)} \in T_{2 l} \text { and } \sigma^{(k)} \geq \sigma^{\left(p_{m}\right)}\right\}
$$

By a counting argument similar to that in the proof of Note 1 we have that 


$$
\sum_{\sigma^{(k)} \in \Delta_{r}} \mu\left(\sigma^{\left(p_{m}\right)}, \sigma^{(k)}\right) f\left(\prod_{i=1}^{2 l} x_{i}^{\sigma^{(k)}(i)}\right)=(-1)^{d_{r}-d_{m}} f\left(x_{r}\right)
$$

where $d_{r}$ and $d_{m}$ are the number of $i$ 's such that $x^{(i)}=\beta^{(i)}$ in $x_{r}$ and $x_{m}$ respectively. Thus $L\left(X, \sigma^{\left(p_{m}\right)}\right) f=\Delta_{f} I_{m}$.

Note 3. Let $f: I \rightarrow B, I=[\alpha, \beta] \subset R^{l}$ and $X=\left\{x_{1}, x_{2}, \ldots, x_{2} l\right\}$ with $X$ the vertices of $I$ numbered as in Note 1 . For $\Gamma_{m}$ as in (1) and $\sigma^{\left(p_{m}\right)} \in \Gamma_{m}$, $\sigma^{\left(p_{m}\right)} \geq \sigma^{(k)}$ for all $\sigma^{(k)} \in \Gamma_{m}$ we have that $L\left(X, \sigma^{(k)}\right) f=0$ for $k \neq p_{m}$.

Proof. Suppose $\sigma^{(j)} \in \Gamma_{m}, \sigma^{(j)}<\sigma^{\left(p_{m}\right)}$.

$$
\begin{aligned}
L\left(X, \sigma^{(j)}\right) f= & \sum_{\sigma(k) \in T_{2} l} \mu\left(\sigma^{(j)}, \sigma^{(k)}\right) f\left(\prod_{i=1}^{2 l} x_{i}^{\sigma^{(k)}(i)}\right) \\
= & \sum_{\sigma \in \Gamma_{m}} \mu\left(\sigma^{(j)}, \sigma\right) f\left(\prod_{i=1}^{2 l} x_{i}^{\sigma(i)}\right)+\sum_{\sigma \in \Gamma_{m}(1)} \mu\left(\sigma^{(j)}, \sigma\right) f\left(\prod_{i=1}^{2 l} x_{i}^{\sigma(i)}\right) \\
& +\cdots+\sum_{\sigma \in \Gamma_{m}(s)} \mu\left(\sigma^{(j)}, \sigma\right) f\left(\prod_{i=1}^{2 l} x_{i}^{\sigma(i)}\right)
\end{aligned}
$$

such that $x_{m_{i}}-x_{m} \in Q_{1}$ for $i=1,2, \ldots$, s. Now $\Sigma_{\sigma \epsilon} \mu\left(\sigma^{(j)}, \sigma\right) f\left(\Pi_{i=1}^{2 l} x_{i}^{\sigma(i)}\right)$ contains $2^{r}$ nonzero summands where $r$ is the number of $i$ 's such that $\sigma^{\left(p_{m}\right)}(i)-\sigma^{(j)}(i)=1$ and $2^{r-1}$ of them are such that $\mu\left(\sigma^{(j)}, \sigma\right)=+1$ and $2^{r-1}$ are such that $\mu\left(\sigma^{(j)}, \sigma\right)=-1$. Hence

$$
\sum_{\sigma \in \mathbf{\Gamma}_{m}} \mu\left(\sigma^{(j)}, \sigma\right) f\left(\prod_{i=1}^{2 l} x_{i}^{\sigma(i)}\right)=0 .
$$

Also $\Sigma_{\sigma \in \Gamma_{m(k)}} \mu\left(\sigma^{(j)}, \sigma\right) f\left(\Pi_{i=1}^{2 l} x_{i}^{\sigma(i)}\right)$ contains $2^{s}$ summands where $s$ is the number of $i$ 's such that $\sigma^{\left(p_{m}(k)\right)}(i)-\sigma^{(j)}(i)=1$ and again $2^{s-1}$ of them are such that $\mu\left(\sigma^{(j)}, \sigma\right)=+1$ and $2^{s-1}$ are such that $\mu\left(\sigma^{(j)}, \sigma\right)=-1$ so that

$$
\sum_{\sigma \in \Gamma_{m}(i)} \mu\left(\sigma^{(j)}, \sigma\right) f\left(\prod_{i=1}^{r} x_{i}^{\sigma(i)}\right)=0
$$

and the conclusion holds.

Lemma 3.5. Let $f: l \rightarrow B, I=[\alpha, \beta] \subset R^{l}$ and $X=\left\{x_{1}, x_{2}, \ldots, x_{2 l}\right\}$ be the vertices of $I$ with $x_{1}=\alpha$ and $x_{2 l}=\beta$. Then

$$
\sum_{\sigma \in T_{2} l}\|L(X, \sigma) f\|=\left\|\Delta_{f} I\right\|+\sum_{J \in \Omega}\left\|\Delta_{f} J\right\|
$$


Proof. By definition

$$
\sum_{\sigma \in T_{2 l}}\|L(x, \sigma) f\|=\sum_{n=1}^{2 l}\left\|L\left(X, \sigma^{\left(p_{m}\right)}\right) f\right\|+\sum_{\sigma^{(k) \neq \sigma^{\left(p_{m}\right)}}}\left\|L\left(X, \sigma^{(k)}\right) f\right\| .
$$

By Note $3 L\left(X, \sigma^{(k)}\right) f=0$ for $k \neq p_{m}$ for some $m$ so that

$$
\sum_{\sigma \in T_{2} l}\|L(X, \sigma) f\|=\sum_{m=1}^{2 l}\left\|L\left(X, \sigma^{\left(p_{m}\right)}\right) f\right\| .
$$

But by Note $1, L\left(X, \sigma^{2^{\left(2^{l}-1\right)}}\right) f=\Lambda_{f} I$ and by Note $2, L\left(X, \sigma^{\left(p_{m}\right)}\right) f=\Delta_{f} I_{m}$ so the lemma holds.

The following lemmas yield the equivalence of $V_{2}(f ; l)$ and $V_{4}(f ; I)$ provided $V_{2}(f ; J)=0$ for $J \in \Omega$.

Lemma. Let $f: I \rightarrow B, I=[\alpha, \beta] \subset R^{l}$. Let $X=\left\{x_{1}, x_{2}, \ldots, x_{n}\right\}$ be a partition of $I$ and $X_{0}=X \cup\{y\}, y \notin X$, be a second partition of $I$. Then

$$
\sum_{\sigma \in T_{n}}\|L(X, \sigma) f\| \leq \sum_{\sigma \in T_{n+1}}\left\|L\left(X_{0}, \sigma\right) f\right\| .
$$

This lemma follows since $L\left(X, \sigma^{(k)}\right) f=L\left(X_{0}, \tau_{1}\right) f+L\left(X_{0}, \tau_{2}\right) f$ where

$$
\tau_{1}(i)= \begin{cases}\sigma^{(k)}(i) & \text { for } i<m, \\ 0 & \text { for } i=m, \\ \sigma^{(k)}(i-1) & \text { for } i>m,\end{cases}
$$

and

$$
\tau_{2}(i)= \begin{cases}\tau_{1}(i) & \text { for } i \neq m, \\ 1 & \text { for } i=m .\end{cases}
$$

Lemma 3.6. Let $f: I \rightarrow B, I=[\alpha, \beta] \subset R^{l}$ and $X=\left\{x_{1}, x_{2}, \ldots, x_{r}\right\}$ be a regular partition of $I$ with $\left\{I_{1}, I_{2}, \ldots, I_{s}\right\}$ the corresponding regular subdivision of $I$ in the calculation of $V_{4}(f ; l)$. Then

$$
\sum_{\sigma \in T_{r}}\|L(X, \sigma) f\|=\sum_{m=1}^{s}\left\|\Delta_{f} I_{m}\right\|
$$

assuming the variation, $V_{2}(f ; J)=0$ for $J \in \Omega$.

The proof follows in a manner similar to that of Note 1.

Lemma 3.7. Let $f: I \rightarrow B, I=[\alpha, \beta] \subset R^{l}$ and $V_{2}(f ; J)=0$ for $J \in \Omega$. Then $f \in B V_{2}$ if and only if $f \in B V_{4}$. 
This follows from lemmas above.

It remains to establish a relationship between $V_{1}$ and either $V_{2}$ or $V_{4}$. Note 4. Let $f: I \rightarrow B, I=[\alpha, \beta] \subset R^{l}, f \in B V_{2}$ and $V_{2}(f ; l)=0$ for $J \in \Omega$. Then $f \in B V_{1}$.

Proof. From Lemma 3.7, $B V_{2}$ and $B V_{4}$ are equivalent when $V_{2}(f ; J)=0$ for $J \in \Omega$. The calculation of $V_{4}$ is symmetrical and the calculations of $V_{2}$ and $V_{1}$ agree when the partition is ordered by $Q_{1}$ so the result follows.

The converse of Note 4 does not hold. Consider the function $f: I \rightarrow R, I=$ $[0,1] \subset R^{2}$ defined by

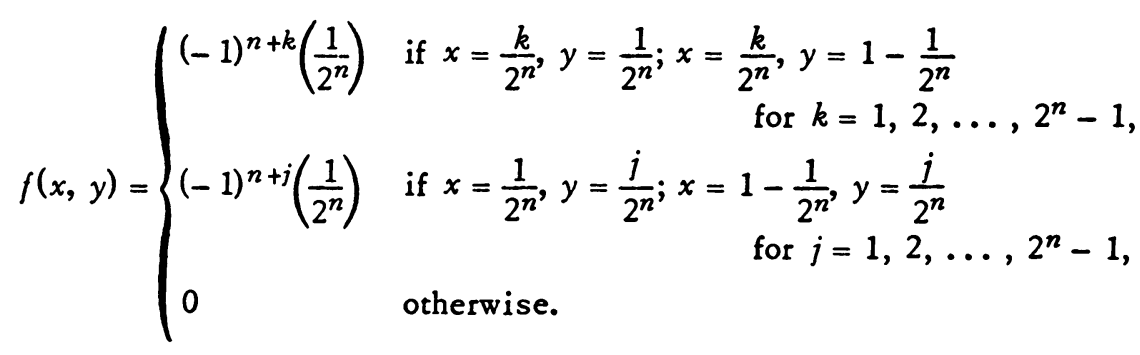

For $N$ any positive integer, the regular partition $P=\left\{\left(k / 2^{N}, 1 / 2^{N}\right): k, j=\right.$ $\left.0,1,2, \ldots, 2^{N}\right\}$ we have that

$$
\begin{aligned}
V_{2}(f ; l) & \geq \sum_{j=0}^{2^{N}} \sum_{k=0}^{2^{N}}\left|f\left(\frac{k}{2^{N}}, \frac{j}{2^{N}}\right)-f\left(\frac{k+1}{2^{N}}, \frac{j}{2^{N}}\right)-f\left(\frac{k}{2^{N^{N}}}, \frac{j+1}{2^{N}}\right)+f\left(\frac{k+1}{2^{N}}, \frac{i+1}{2^{N}}\right)\right| \\
& \geq \frac{1}{2}+2+3(N-2)
\end{aligned}
$$

since

$$
f\left(\frac{k}{2^{N}}, \frac{j}{2^{N}}\right) f\left(\frac{k+1}{2^{N}}, \frac{j+1}{2^{N}}\right) \geq 0, \quad f\left(\frac{k+1}{2^{N}}, \frac{j}{2^{N}}\right) f\left(\frac{k}{2^{N}}, \frac{j+1}{2^{N}}\right) \geq 0
$$

and

$$
f\left(\frac{k}{2^{N}}, \frac{j}{2^{N}}\right) f\left(\frac{k+1}{2^{N}}, \frac{j}{2^{N}}\right) \leq 0
$$

Hence $V_{2}(f ; I)>N$ for $N \geq 2$.

On the other hand for any partition $P \in \mathcal{P}, \mathcal{P}$ the set of all finite nonempcy partitions linearly ordered by either $Q_{1}$ or $Q_{2}$, we have $V_{1}(f ; l) \leq$ $\left[2\left(2^{N}\right)-1\right]\left(1 / 2^{N}\right) \leq 2$ since $P$ contains at most $2\left(2^{n}\right)-1$ points of the form $\left(k / 2^{n}\right.$. $\left.j / 2^{n}\right)$ with a total number less than or equal to $2\left(2^{N}\right)-1$ so that $f \in B V_{1}$.

The example showing that $f \in B V_{3}$ does not imply $f \in B V_{1}$ also shows 
then that $f \in B V_{3}$ does not imply $f \in B V_{2}$ or $f \in B V_{4}$.

4. Primary results. We now address the question of giving conditions which when satisfied by the sequence $\left\{y_{k}\right\}, y_{k} \in \Pi_{s \in S} E_{s}$, where $S$ is a nonempty set in $R^{l}$ and each $E_{s}$ is an ordered vector space, guarantee the existence of a subsequence of $\left\{y_{k}\right\}$ which converges pointwise on $S$. With this in mind we make the following definition.

Definition 4.1. Let $S$ be a nonempty subset of $R^{l}$ and $f$ be a function, $f \in \Pi_{s \in S} E_{s}$ where each $E_{s}$ is an ordered vector space with positive cone $K_{s}$. Consider the set $\mathcal{P}$ of all finite nonempty partitions $P=\left\{x_{1}, x_{2}, \ldots, x_{n}\right\}$ of $S$ where $n \geq 1, x_{i} \in S$ for $i=1,2, \ldots, n$ and $P$ is linearly ordered by some positive cone $Q_{j}$ for $j=1,2, \ldots, 2^{(l-1)}$. If $f(s) \neq \theta_{s}$ for some $s \in S$ we say that $(f, P)$ is a proper pair if $(-1)^{i} f\left(x_{i}\right)>\theta_{x_{i}}$ for $i=1,2, \ldots, n$ or else $(-1)^{i} f\left(x_{i}\right)<\theta_{x_{i}}$ for $i=1,2, \ldots, n$. If $f(s)=\theta_{s}$ for all $s \in S$ we say that $(f, P)$ is a proper pair if $P$ contains exactly one point.

We will say that the sequence is an eventually comparable sequence if there exists a positive integer $M(t)$ such that $y_{k}(t)$ and $y_{j}(t)$ are comparable for $k, j \geq M(t)$.

Theorem 4.2. Let $S$ be a nonempty subset of $R^{l}$ and $\left\{y_{k}\right\}$ be a sequence of functions, $y_{k} \in \Pi_{s \in S} E_{s}$, where each $E_{s}$ is an ordered vector space with positive cone $K_{s}$. For each $t \in S$ assume that $\left\{y_{k}(t)\right\}$ is an eventually comparable sequence and that there is a number $\delta(t) \geq 0$ and positive integers $N(t)$ and $H(t)$ such that for each $k, j \geq H(t)$ the partitions $P \in \mathcal{P}$ such that $\left(y_{k}-y_{j}, P\right)$ is a proper pair, each contain at most $N(t)$ points in the sphere $D(t, \delta(t))$. Then $\left\{y_{k}\right\}$ contains a subsequence $\left\{h_{k}\right\}$ such that $\left\{h_{k}(t)\right\}$ is eventually monotone for each $t \in S$.

Proof. If $y_{k}(t)$ and $y_{j}(t)$ are comparable for all $k, j \geq M(t)$ and $M(t)$ is the smallest integer having this property we let $A_{i}=\{t: t \in S, M(t)=i\}$ for $i=1,2, \ldots$. For any $z \in A_{i}$ we have $y_{k}(t)$ and $y_{j}(t)$ comparable for $k, j \geq$ $i$. We will prove the theorem assuming that $y_{k}(t)$ and $y_{j}(t)$ are comparable for all $t \in S$ and then a standard disagonalization argument where $S$ is replaced by $A_{1}, A_{2}, \ldots$ yields the desired result.

We note that we may assume $S$ is bounded because if the theorem is true for bounded sets a standard diagonalization argument with $S \cap D(0,1), S \cap$ $D(0,2), \ldots$ yields the result for unbounded sets. Also, we may assume $S$ is a closed interval because if the theorem is true for closed intervals $I=[\alpha, \beta]$, then we may choose $I$ to be a closed interval containing the bounded set $S$ and define a sequence of functions $\left\{Z_{k}\right\}, Z_{k} \in \Pi_{s \in I} F_{s}$ where $F_{s}=E_{s}$ for $s \in S$ and $F_{s}=R$ (with the usual order) for $s \in I, s \notin S$, by 


$$
Z_{k}(t)= \begin{cases}y_{k}(t) & \text { for } t \in S, \\ 0 & \text { for } t \notin S\end{cases}
$$

Then the sequence $\left\{Z_{k}\right\}$ satisfies the hypotheses of the theorem on $I$ since for $\left(Z_{k}-Z_{j}, P\right)$ a proper pair either $Z_{k}(t)-Z_{j}(t)=0$ for all $t \in I$ or else $P$ contains no points in $I-S$. Also, since we may assume $S$ is a compact interval there exists a finite number of $t_{i} \in S, i=1,2, \ldots, n$, such that $D\left(t_{i}, \delta\left(t_{i}\right)\right)$, $i=1,2, \ldots, n$, cover $S$ and by choosing $H=\max _{1 \leq i \leq n}\left\{H\left(t_{i}\right)\right\}$ and $N=$ $\sum_{i=1}^{n} N\left(t_{i}\right)$ we have integers $N$ and $H$ such that for $k, j \geq H$ the partitions $P \in \mathcal{P}$ such that $\left(y_{k}-y_{j}, P\right)$ is a proper pair, each contain at most $N$ points in $S$. Hence without loss of generality we may assume $H=1$.

The theorem will now be proved by induction on $l$ for $S$ a compact interval in $R^{l}$.

Let $l=1, S$ be a compact interval and $\left\{y_{k}\right\}$ be a sequence of functions $y_{k} \in \Pi_{s \in S} E_{s}$ where $E_{s}$ is an ordered vector space with positive cone $K_{s}$. For each $k, j$ and $t \in S$ assume that $y_{k}(t)$ is comparable with $y_{j}(t)$. Let $N$ be a positive integer such that for each $k, j$ the partitions $P \in \mathcal{P}$ with $\left(y_{k}-y_{j}, P\right)$ a proper pair each contain at most $N$ points in $S$. The case for $l=1$ will now be proved by induction on $N$. If $N=1$ then for each $k, j$ either $y_{k}(t)-y_{j}(t) \in K_{t}$ holds for all $t \in S$ or else $y_{j}(t)-y_{k}(t) \in K_{t}$ holds for all $t \in S$. Thus by the mapping $g$, defined in the proof of Corollary 2.2, it is possible to pick a subsequence of $\left\{y_{k}\right\}$ that is monotone on $S$. Now assume the theorem holds for $N=1,2, \ldots, K$. We will then show this implies it is correct for $N=K+1$.

If there are only finitely many functions in $\left\{y_{k}\right\}$ which are distinct on $S$ then infinitely many are identical on $S$ and using that as the subsequence we are done. Thus we may assume there are infinitely many functions in $\left\{y_{k}\right\}$ which are distinct on $S$ and, by picking a subsequence if necessary, we may assume all the $y_{k}$ are distinct on $S$.

Let $I, J$ be subintervals of $S$ with $I \cup J=S, I \cap J=\varnothing$ and let $I$ and $J$ be of the same length. We will show that there is a subsequence of $\left\{y_{k}\right\}$ that is eventually monotone for each $t \in I$ or else is eventually monotone for each $t \in J$. Now let $\Gamma=\left\{y_{k}\right\}, u=K+1$ and $r=2$ with $C_{1}=\left\{\left\{y_{k}, y_{j}\right\}: k \neq j\right.$, proper pairs $\left(y_{k}-y_{j}, P\right)$ are such that $P$ has at most one point $\left.x \in I\right\}$. Now for $n=2,3, \ldots, K+1$ let $C_{n}=\left\{\left\{y_{k}, y_{j}\right\}: k \neq j\right.$, proper pairs $\left(y_{k}-y_{j}, P\right)$ are such that $P$ has at most $n$ points $x_{1}, x_{2}, \ldots, x_{n} \in I$ and $\left\{y_{k^{\prime}} y_{j}\right\} \notin C_{n-p}$, $p=1,2, \ldots, n-1\}$. By Theorem 2.1 there is an infinite subclass $\Delta$ of $\Gamma$ such that all pairs of elements of $\Delta$ belong to the same $C_{m}$ for some $m=$ $1,2, \ldots, K+1$. If $m=1,2, \ldots, K$ then the induction hypothesis holds on 
$I$ and there is a subsequence $\left\{h_{j}\right\}$ of $\left\{y_{k}\right\}$ that is eventually monotone at each point of $I$. Note that if $\left\{y_{k}, y_{j}\right\} \in C_{m}$ then proper pairs $\left(y_{k}-y_{j}, P\right)$ are such that $P$ has at most $K+2-m$ points $x_{1}, x_{2}, \ldots, x_{K+2-m} \in J$ so that if $m=2,3, \ldots, K$ a similar argument shows that the induction hypothesis holds on $J$ so there is a subsequence $\left\{g_{i}\right\}$ of $\left\{h_{j}\right\}$ that is eventually monotone at each point of $l \cup J=S$ and the case $l=1$ holds. If $m=K+1$ then the proper pairs $\left(y_{k}-y_{j}, P\right)$ are such that $P$ has at most one point $x \in J$ and there is a subsequence $\left\{h_{j}\right\}$ of $\left\{y_{k}\right\}$ that is eventually monotone at each point of $J$. In any case either the case $l=1$ holds or there is a subsequence that is eventually monotone at each point of $I$ or $J$. Let $S_{1}$ denote the interval $I$ or $J$ on which $\left\{h_{j}\right\}$ is not known to be eventually monotone at each point.

We now repeat the entire process described in the preceding paragragh on $S_{1}$ obtaining $S_{2}$, then on $S_{2}$ obtaining $S_{3}$, etc. If the theorem does not hold at any step in the induction, use the standard diagonalization argument to obtain a sequence $\left\{h_{j}\right\}$. Let $x_{0}$ be the unique limit point of the set of midpoints of the intervals $S_{n}$. Choose $\left\{g_{i}\right\}$ to be a subsequence of $\left\{h_{j}\right\}$ such that $\left\{g_{i}\left(x_{0}\right)\right\}$ is monotone so $\left\{g_{i}\right\}$ now has the property that it is eventually monotone at each point of $S$ and the case for $l=1$ holds.

Assume the theorem is true for $l=L-1$ so it remains to show that it holds for $l=L$.

Let $S=[\alpha, \beta]$ be a compact interval in $R^{L}$ and $\left\{y_{k}\right\}$ be a sequence of functions, $y_{k} \in \Pi_{s \in S} E_{s}$, where each $E_{s}$ is an ordered vector space with positive cone $K_{s}$. For each $k, j$ and $t \in S$ assume that $y_{k}(t)$ is comparable with $y_{j}(t)$. Let $N$ be a positive integer such that for each $k, j$ the partitions $P \in \mathscr{P}$ with $\left(y_{k}-y_{j}, P\right)$ a proper pair each contain at most $N$ points in $S$. Now the case for $l=L$ will be proved by induction on $N$. If $N=1$ then for each $k, j$ either $y_{k}(t)-y_{j}(t) \in K_{t}$ holds for all $t \in S$ or else $y_{j}(t)-y_{k}(t) \epsilon$ $K_{t}$ holds for all $t \in S$. Thus it is possible to pick a subsequence as in the proof of Corollary 2.2 which is a monotone sequence on $S$. We now assume the theorem holds for $N=1,2, \ldots, K$ and will show this implies it is correct for $N=K+1$.

If there are only finitely many functions in $\left\{y_{k}\right\}$ which are distinct on $S$ then infinitely many are identical on $S$ and we are done. Thus we may assume there are infinitely many functions in $\left\{y_{k}\right\}$ that are distinct on $S$ and, by picking a subsequence if necessary, we may assume all the $y_{k}$ 's are distinct. Let $I, J$ be subintervals of $S$ with $I \cup J=S, I \cap J=\varnothing, I=\left[\alpha, \gamma_{1}\right]-X, J=$ $\left[\gamma_{2}, \beta\right]$ where $\gamma_{1}=\left(1 / 2\left(\alpha^{(1)}+\beta^{(1)}\right), \beta^{(2)}, \beta^{(3)}, \ldots, \beta^{(L)}\right), \gamma_{2}=\left(1 / 2\left(\alpha^{(1)}+\beta^{(1)}\right)\right.$, $\left.\alpha^{(2)}, \alpha^{(3)}, \ldots, \alpha^{(L)}\right)$ and $x_{0}=\left\{\left(1 / 2\left(\alpha^{(1)}+\beta^{(1)}\right), x^{(2)}, \ldots, x^{(L)}\right): \alpha^{(i)} \leq x^{(i)}\right.$ 
$\leq \beta^{(i)}$ for $\left.i=2,3, \ldots, L\right\}$. We will show by the same argument as for $l=1$ that there is a subsequence of $\left\{y_{k}\right\}$ that is eventually monotone for each $t \epsilon$ $I$ or else is eventually monotone for each $t \in J$. Since all $y_{k}(\beta)$ are comparable there is a subsequence of $\left\{y_{k}\right\}$, denoted again by $\left\{y_{k}\right\}$, such that $\left\{y_{k}(\beta)\right\}$ is monotone. Now using Theorem 2.1 with $\Gamma=\left\{y_{k}\right\}, u=K+1$ and $r=2$ where $C_{1}=\left\{y_{k}, y_{j}\right\}: k \neq j$, proper pairs $\left(y_{k}-y_{j}, P\right)$ are such that $P$ contains at most one point $x \in I\}$ and $C_{n}=\left\{\left\{y_{k}, y_{j}\right\}: k \neq j\right.$, proper pairs $\left(y_{k}-\right.$ $\left.y_{j}, P\right)$ are such that $P$ has at most $n$ points $x_{1}, x_{2}, \ldots, x_{n} \in I$ and $\left\{y_{k^{\prime}} y_{j}\right\}$ $\left.\notin C_{n-p}, p=1,2, \ldots, n-1\right\}$ for $n=2, \ldots, K+1$ there is an infinite subclass $\Delta$ of $\Gamma$ such that all pairs of elements of $\Delta$ belong to the same $C_{m}$ for some $m=1,2, \ldots$, $K+1$. If $m=1,2, \ldots, K$ then the induction hypothesis holds on $I$ and there is a subsequence $\left\{h_{j}\right\}$ of $\left\{y_{k}\right\}$ such that $\left\{h_{j}\right\}$ is monotone at each point of $I$. Again if $\left\{y_{k}, y_{j}\right\} \in C_{m}$ then the proper pairs $\left(y_{k}-y_{j}, P\right)$ are such that $P$ has at most $K+2-m$ points $x_{1}, x_{2}, \ldots, x_{K+2-m} \in J$. So if $m=2,3, \ldots, K$ then the induction hypothesis holds on $J$ so there is a subsequence $\left\{g_{i}\right\}$ of $\left\{h_{j}\right\}$ that is monotone at each point of $I \cup J=S$ and the theorem holds. If $m=K+1$ then the proper pairs $\left(y_{k}-y_{j}, P\right)$ are such that $P$ has at most one point $x \in J$ and there is a subsequence $\left\{h_{j}\right\}$ of $\left\{y_{k}\right\}$ that is monotone at each point of $J$. In any case either the theorem holds or there is a subsequence that is monotone at each point of $I$ or is monotone at each point of $J$. Let $S_{1}$ denote the interval $I$ or $J$ on which $\left\{h_{j}\right\}$ is not known to be eventually monotone.

Now repeat the entire process described in the preceding paragraph on $S_{1}$ to obtain $S_{2}$ so that we obtain a sequence that is eventually monotone on $\left(S-S_{2}\right)$. Continuing, we repeat the entire process on $S_{2}$ to obtain $S_{3}$, on $S_{3}$ to obtain $S_{4}$, etc. If the theorem does not hold at any step in the induction, then by a standard diagonalization argument we obtain a sequence $\left\{h_{j}\right\}$ that is eventually monotone on $S_{0}=\left(S-\bigcap_{j=1}^{+\infty} S_{i}\right)$. But. $\bigcap_{i=1}^{+\infty} S_{i}$ is an $L-1$ dimensional interval so by the induction hypothesis on the dimension of the domain it is possible to choose $\left\{g_{i}\right\}$, a subsequence of $\left\{h_{j}\right\}$, such that $\left\{g_{i}(x)\right\}$ is eventually monotone for $x \in \bigcap_{i=1}^{+\infty} S_{i}$. Now $\left\{g_{i}\right\}$ has the property that it is eventually monotone at each point of $S \subset R^{L}$ so the conclusion of the theorem holds.

In the next theorem, by further restricting $E_{s}$, we obtain a subsequence $\left\{h_{j}\right\}$ of $\left\{y_{k}\right\}$ such that $\left\{h_{j}(s)\right\}$ is eventually monotone in $E_{s}$ or else converges in $E_{s}$ for each $s \in S$. The proof will again be done by induction on the dimension of the domain. The following is the case for $l=1$.

Theorem 4.3. Let $S$ be a nonempty subset of real numbers and $\left\{y_{k}\right\}$ be a sequence of functions, $y_{k} \in \Pi_{s \in S} E_{s}$ where each $E_{s}$ is a sequentially com- 
plete ordered locally convex space with positive cone $K_{s^{*}}$. For each $t \in S$ assume that $\left\{y_{k}(t)\right\}$ is an eventually comparable sequence. Assume $E_{s}$ has a nested countable basis of circled sets at $\theta_{s}$ denoted by $\left\{U_{s}(n)\right\}$. For each $t \in S$ and each positive integer $n$ assume that there are nonnegative integers $N(n, t), H(n, t)$ and a number $\delta(n, t)>0$ such that for all $k, j \geq H(n, t)$ if $\left(y_{k}-y_{j}, P\right)$ is a proper pair then $P$ contains at most $N(n, t)$ points $x$ such $y_{k}(x)-y_{j}(x) \notin U_{x}(n)$ and $t-\delta(n, t)<x<t+\delta(n, t)$. Then $\left\{y_{k}\right\}$ contains $a$ subsequence $\left\{h_{j}\right\}$ such that for each $t \in S,\left\{h_{j}(t)\right\}$ is either eventually monotone or else is convergent.

\section{Proof. See note following Theorem 2.2 in [15].}

Now for the case $l>1$ the following theorem yields the desired conclusion.

Theorem 4.4. Let $S$ be a nonempty subset of $R^{l}$ and $\left\{y_{k}\right\}$ be a sequence of functions, $y_{k} \in \Pi_{s \in S} E_{s}$, where each $E_{s}$ is a sequentially complete ordered locally convex space with positive cone $K_{s}$. For each $t \in S$ assume that $\left\{y_{k}(t)\right\}$ is an eventually comparable sequence: Assume $E_{s}$ has a nested countable basis of circled sets of $\theta_{s}$ denoted by $\left\{U_{s}(n)\right\}$. For each $t \in S$ and each positive integer $n$ assume that there are nonnegative integers $N(n, t)$, $H(n, t)$ and a number $\delta(n, t)>0$ such that for all $k, j>H(n, t)$ if $\left(y_{k}-y_{j}, P\right)$ is a proper pair then $P$ contains at most $N(n, t)$ points $x$ such that $y_{k}(x)$ $y_{j}(x) \notin U_{x}(n)$ and $x$ is in the sphere $D(t, \delta(n, t))$. Then $\left\{y_{k}\right\}$ contains a sub. sequence $\left\{h_{k}\right\}$ such that for each $t \in S,\left\{h_{k}(t)\right\}$ is either eventually monotone or else is convergent.

Proof. As in the proof of Theorem 3.1 we may assume that $y_{k}(t)$ and $y_{j}(t)$ are comparable for all $k, j$ and $t \in S$. Also we will again assume $S$ is a bounded closed interval, $S=[\alpha, \beta]$ and $H(n, t)$ will be replaced by $H(n)$ and $N(n)$ respectively.

The proof now will be by induction on the dimension of the domain. The case for $l=1$ is Theorem 3.2. Assume the result holds for $l=K-1$ then we will show that the theorem is true for $l=K$.

Let $\left\{J_{i}\right\}$ be an enumeration of the spheres $D(t, \delta) \subset S$ in $R^{K}$ with rational centers and rational radii.

Now for $J_{1}$ by Corollary 2.2 there is a subsequence of $\left\{y_{k}\right\}$, again denoted $\left\{y_{k}\right\}$, that is monotone on $J_{1}$ or else there is a subsequence of $\left\{y_{k}\right\}$ again denoted by $\left\{y_{k}\right\}$ such that for $k \neq j, y_{k}(t)>y_{j}(t)$ for some $t \in J_{1}$ and $y_{k}(\tau)<$ $y_{j}(\tau)$ for some $\tau \in J_{1}$. Now repeat the process described in the preceding sentence for $J_{2}, J_{3}, \cdots$ and then take the diagonal subsequence, denote it again by $\left\{y_{k}\right\}$. This sequence has the property that on $\left\{J_{i}\right\}$ it is either eventually 
monotone or else for every $k \neq j$ sufficiently large, depending on $i$, there exists $t, \tau \in J_{i}$ such that $y_{k}(t)>y_{j}(t)$ and $y_{k}(\tau)<y_{j}(\tau)$.

Now using $J_{1}$ and $U_{t}(1)$ by Corollary 2.3 there exists a subsequence of $\left\{y_{k}\right\}$, again denoted by $\left\{y_{k}\right\}$, such that, for $k \neq j, y_{k}(t)-y_{j}(t) \in U_{t}(1)$ for all $t \in J_{1}$ or else there is a subsequence of $\left\{y_{k}\right\}$, again denoted by $\left\{y_{k}\right\}$, such that, for $k \neq j$ there is a $t \in J_{1}$ with $y_{k}(t)-y_{j}(t) \notin U_{t}(1)$. Now repeat the process described in the previous sentence using $U_{t}(2), U_{t}(3), \ldots$ and then take the diagonal subsequence and denote it again by $\left\{y_{k}\right\}$. This sequence has the property that for $J_{1}$ and $U_{t}(n)$ either for all $k \neq j$ sufficiently large, depending on $n, y_{k}(t)-y_{j}(t) \in U_{t}(n)$ for all $t \in J_{1}$ or else for all $k \neq j$ sufficiently large depending on $n$, there is some $t \in J_{1}$ such that $y_{k}(t)-y_{j}(t)$ $\notin U_{t}(n)$.

We now repeat the entire process described in the preceding paragraph consecutively on the spheres $J_{2}, J_{3}, \cdots$ and then take the diagonal subsequence again denoted by $\left\{y_{k}\right\}$. This sequence has the property that for $J_{i}$ and $U_{t}(n)$ either for $k \neq j$ sufficiently large depending on $i$ and $n, y_{k}(t)-y_{j}(t) \epsilon$ $U_{t}(n)$ for every $t \in J_{i}$ or else for every $k \neq j$ sufficiently large, depending on $i$ and $n$, there exists a $t \in J_{i}$ such that $y_{k}(t)-y_{j}(t) \notin U_{t}(n)$.

Let $\Gamma_{1}, \Gamma_{2}, \ldots, \Gamma_{2 K}$ be the bounding edges of $S$; then $\Gamma_{1}$ is a $K-1$ dimensional interval so by the induction hypothesis there is a subsequence of $\left\{y_{k}\right\}$, denote it again by $\left\{y_{k}\right\}$, such that for $x \in \Gamma_{1},\left\{y_{k}(x)\right\}$ is either eventually monotone or else is convergent. Then by similarly extracting a subsequence from $\left\{y_{k}\right\}$ on $\Gamma_{2}, \Gamma_{3}, \ldots, \Gamma_{2 K}$ consecutively we obtain a subsequence that is either eventually monotone or convergent for each point of the bounding edges of $S$.

Now suppose $A_{0}$ is the set of $x$ 's in $S$ such that $\left\{y_{k}(x)\right\}$ does not converge and is not eventually monotone. If $A_{0}$ is countable then by a standard diagonalization argument there is a subsequence of $\left\{y_{k}\right\}$, denote it again by $\left\{y_{k}\right\}$, that is either eventually monotone or else is convergent for each $x \in S$. So suppose $A_{0}$ is uncountable. Let $\Gamma$ be the set of $K-1$ dimensional hyperplanes of the form $\gamma=\left\{\left(x^{(1)}, x^{(2)}, \ldots, x^{(K)}\right): x^{(i)}=c\right.$ for $i$ fixed, $\alpha^{(i)} \leq$ $\left.c \leq \beta^{(i)}\right\}$ which have nonempty intersection with $A_{0}$. Consider the set $\Psi$ of sets $B$ of the form

$$
B=\left\{\left(\gamma, a_{\gamma}\right): \gamma \in \Gamma, a_{\gamma} \in A_{0} \cap \gamma, a_{\gamma_{1}}-a_{\gamma_{2}} \in \bigcup_{i=1}^{2 K-1}\left[Q_{i}^{0} \cup\left(-Q_{i}^{0}\right)\right\} .\right.
$$

Now order the sets $B$ in $\Psi$ by $B_{1} \leq B_{2}$ if and only if $B_{1} \subset B_{2}$. Then the union of any linearly ordered chain in $\Psi$ is an upper bound of the chain and is again in $\Psi$ so by Zorn's lemma there must be a maximal element $B_{0}$ in $\Psi$.

If $B_{0}$ is uncountable and $\left(\gamma_{1}, a_{\gamma_{1}}\right),\left(\gamma_{2}, a_{\gamma_{2}}\right)$ are in $B_{0}$ then $\left(a_{\gamma_{1}}-\right.$ 
$\left.a_{\gamma_{2}}\right) \in \bigcup_{i=1}^{2 K-1}\left[Q_{i}^{0} \cup\left(-Q_{i}^{0}\right)\right]$. Let $A_{1}=\left\{a_{\gamma}:\left(\gamma, a_{\gamma}\right) \in B_{0}\right\}$. Now for $x \in A_{1}$ let $\left\{F_{x i}\right\}$ be the subsequence of $\left\{J_{i}\right\}$ consisting of the spheres which contain

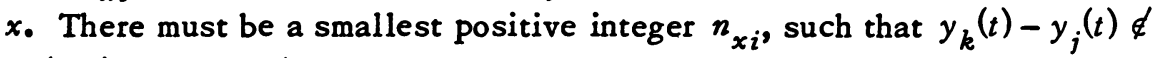
$U_{t}\left(n_{x i}\right)$ for all $k \neq j$ sufficiently large, depending on $i$, for some $t \in F_{x i}$ for otherwise $\left\{y_{k}\right\}$ would be Cauchy on $F_{x i}$ and hence would be convergent at $x$ which is contrary to the choice of $x$. If $\overline{\lim }_{i \rightarrow+\infty} n_{x i}=+\infty$ then there is a subsequence $\left\{n_{x i(\alpha)}\right\}$ of $\left\{n_{x i}\right\}$ such that $\lim _{\alpha \rightarrow+\infty} n_{x i(\alpha)}=+\infty$ and by the definition of $n_{x i(a)}$ and the nestedness of $\left\{U_{t}(n)\right\}$ we have that $y_{k}(t)-y_{j}(t) \epsilon$ $U_{t}\left(n_{x i(a)}-1\right)$ for all $k \neq j$ sufficiently large, depending on $\alpha$, and all $t \epsilon$ $F_{x i(a)^{\circ}}$ This implies that $\left\{y_{k}(x)\right\}$ is Cauchy and hence convergent which is contrary to the choice of $x$ so that $\overline{\lim }_{i \rightarrow+\infty} n_{x i}=c_{x}<+\infty$. Let $c_{x}<d_{x}$ be an upper bound for the set $\left\{n_{x i}\right\}$.

Since there are uncountably many values of $x$ in $A_{1}$ at which $\left\{y_{k}(x)\right\}$ is not convergent nor eventually monotone then there is some fixed positive integer $d$ such that $d_{x} \leq d$ holds for uncountably many values of $x$ in $A_{1}$. Call this set $A$ so that $A$ is an infinite set of distinct elements. Let $\Gamma_{0}=A$, $u=2^{K-1}$ and $r=2$ with $C_{m}=\left\{\left\{a_{\gamma_{k}}, a_{\gamma_{j}}\right\}: a_{\gamma_{k}}-a_{\gamma_{j}} \in\left(\left(Q_{m}^{0}\right) \cup\left(-Q_{m}^{0}\right)\right)\right\}$ for $m=1,2,3, \ldots, 2^{K-1}$. Then by Theorem 2.1 there is an infinite subclass $\Delta$ of $\Gamma_{0}$ such that for $x, y \in \Delta, x-y \in\left(-Q_{m}^{0} \cup\left(-Q_{m}^{0}\right)\right)$ for $m$ fixed. Also for $x \in \Delta$ and $k \neq j$ sufficiently large, depending on $i$, there exists a $t \in F_{x i}$ such that $y_{k}(t)-y_{j}(t) \notin U_{t}(d)$.

Choose $N>N(d)$ and $u(1) \in \Delta \cap S^{0}$ and $F_{u(1) i(1)} \in\left\{F_{u(1) i}\right\}$ such that $\left(S-F_{u(1) i(1)}\right) \cap \Delta$ is infinite. Choose $u(2) \in\left(S-F_{u(1) i(1)}\right) \cap\left(\Delta \cap S^{0}\right)$ and $F_{u(2) i(2)} \in\left\{F_{u(2) i}\right\}$ such that $F_{u(1) i(1)} \cap F_{u(2) i(2)}=\varnothing$ and $\left(S-\left(F_{u(1) i(1)}\right.\right.$ $\left.\cup F_{u(2) i(2)}\right) \cap \Delta$ is infinite. Continuing in this manner we get $\{u(1), u(2)$, $\ldots, u(2 N+1)\}$ in $\Delta \cap S^{0}$ and $\left\{F_{u(1) i(1)}, F_{u(2) i(2)}, \ldots, F_{u(2 N+1) i(2 N+1)}\right\}$ which are mutually disjoint. Now by renaming if necessary we may assume that $u(j+1)-u(j) \in Q_{m}^{0}$ for $j=1,2, \ldots, 2 N$. Let

$$
\begin{aligned}
p=\min _{1 \leq i \leq 2 N}\left\{\left|u^{(1)}(i)-u^{(1)}(i+1)\right|,\left|u^{(2)}(i)-u^{(2)}(i+1)\right|\right. \\
\\
\left.\cdots,\left|u^{(K)}(i)-u^{(K)}(i+1)\right|\right\}
\end{aligned}
$$

so $p>0$. Choose $G_{u(j) i(j)} \in\left\{F_{u(j) i}\right\}$ such that $G_{u(j) i(j)} \subset F_{u(j) i(j)}$ and the radius of $G_{u(j) i(j)}$ is less than $p / 2$. Now $\left\{G_{u(1) i(1)}, G_{u(2) i(2,} \ldots\right.$, $\left.G_{u(2 N+1) i(2 N+1)}\right\}$ are mutually disjoint and if $x \in G_{u(j) i(j)} y \in G_{u(j+1) i(j+1)}$ then $y-x \in Q_{m}^{0}$. Choose $k \neq j, k, j>H(d)$, sufficiently large that for each odd positive integer $a, 1 \leq a \leq 2 N+1, y_{k}\left(x_{d}-y_{j}\left(x_{a}\right) \notin U_{x_{a}}(d)\right.$ for some $x_{a}$ $\epsilon G_{u(a) i(a)}$ and for each positive even integer $\alpha, 2 \leq \alpha \leq 2 N, y_{k}\left(t_{\alpha}\right)-y_{j}\left(t_{\alpha}\right)$ 
$<\theta$ holds for some $t_{a} \in G_{u(a) i(a)}$ and $y_{k}\left(\tau_{a}\right)-y_{j}\left(\tau_{\alpha}\right)>\theta$ holds for some $\tau_{a}$ $\epsilon G_{u(a) i(a)}$. Now consider the partition $P_{0}=\left\{\beta_{1}, \beta_{2}, \ldots, \beta_{n}\right\}$ where $\beta_{a}=$ $x_{\alpha}$ if $\alpha$ is odd; $\beta_{a}$ is omitted from $P_{0}$ if $\alpha$ is even and either $y_{k}\left(x_{(\alpha-1)}\right)$ $y_{j}\left(x_{(a-1)}\right)<\theta$ and $y_{k}\left(x_{(a+1)}\right)-y_{j}\left(x_{(a+1)}\right)>\theta$ or the opposite inequalities hold; $\beta_{a}$ is taken to be $t_{a}$ if $y_{k}\left(x_{(a-1)}\right)-y_{j}\left(x_{(a-1)}\right)>\theta$ and $y_{k}\left(x_{(a+1)}\right)-$ $y_{j}\left(x_{(a+1)}\right)>\theta$ and $\beta_{a}$ is taken to be $\tau_{a}$ if $y_{k}\left(x_{(a-1)}\right)-y_{j}\left(x_{(a-1)}\right)<\theta$ and $y_{k}\left(x_{(a+1)}\right)-y_{j}\left(x_{(a+1)}\right)<\theta$. Then the partition $P_{0}$ is such that $\left(y_{k}-y_{j}, P_{0}\right)$ is a proper pair and $y_{k}\left(x_{a}\right)-y_{j}\left(x_{\alpha}\right) \notin U_{x_{a}}(d)$ for $\alpha$ odd, $x_{a} \in P_{0}$, and there are $N+1$ such points $x_{a}$ which is contrary to the hypothesis of the theorem. Hence it cannot be the case that $B_{0}$ is uncountable.

Let $B_{0}=\left\{\left(\gamma_{1}, a_{\gamma_{1}}\right),\left(\gamma_{2}, a_{\gamma_{2}}\right), \ldots\right\}$ and $\rho_{i}, i=1,2, \ldots$ be the countable number of regular $K-1$ dimensional hyperplanes through the points of $a_{\gamma_{1}}, a_{\gamma_{2}}, \ldots$. Suppose $\gamma \in \Gamma$ with $\gamma \neq \rho_{i}, i=1,2, \ldots$ and $a_{\gamma_{i}} \notin \gamma$ for $i=1,2, \cdots$ then $\left[\bigcap_{i=1}^{+\infty}\left(\gamma \cap \tilde{\rho}_{i}\right)\right] \cap A_{0}=\varnothing$ since otherwise $B_{0}$ would not be maximal. Then $A_{0} \subset \bigcup_{i=1}^{+\infty} \rho_{i}$ so apply the induction hypothesis to the sequence $\left\{y_{k}\right\}$ on $\rho_{1}, \rho_{2}, \cdots$ consecutively, take the diagonal subsequence and denote it again by $\left\{y_{k}\right\}$. This sequence has the property that it is either convergent or eventually monotone at every point in $S$.

5. Corollaries and other results. By restricting the range of the sequence of functions so that eventually monotone sequences in $E_{s}$ converge in $E_{s}$ the conclusion of Theorem $4.4 \mathrm{can}$ be changed to read pointwise convergent. The following is an example of such a theorem.

Theorem 5.1. Let $B$ be a reflexive ordered Banach space with normal positive cone $K$ and $S$ be a nonempty subset of $R^{l}$. If $\left\{y_{k}\right\}$ is a sequence of functions that satisfies the hypothesis of Theorem 4.4 and $\left\{y_{k}(s)\right\}$ is a norm bounded set for each $s \in S$ then there is a subsequence of $\left\{y_{k}\right\}$ which converges for every $s \in S$.

Proof. This follows directly from Theorem 4.4.

Theorem 5.2 (Helly selection theorem). Let $\left\{y_{k}\right\}$ be a sequence of functions, $y_{k}: I \rightarrow R, I=[\alpha, \beta] \subset R^{l}$. Suppose there is a positive constant $K$ with $\left\{y_{k}(x)\right\}$ such that $\left|y_{k}(x)\right| \leq K$ for $k=1,2, \ldots$ and $x \in I$ and $V_{1}\left(y_{k} ; I\right) \leq K$ for $k=1,2, \cdots$. Then there is a subsequence $\left\{h_{k}\right\}$ of $\left\{y_{k}\right\}$ that converges pointwise on $I$.

Proof. Since $V_{1}\left(y_{k}-y_{j} ; I\right) \leq V_{1}\left(y_{k} ; I\right)+V_{1}\left(y_{j} ; I\right) \leq 2 K$ the hypotheses of Theorem 5.1 are satisfied and the conclusion holds.

Since $f \in B V_{4}$ and $f \in B V_{2}$ implies $f \in B V_{1}$ the above Helly selection theorem holds if $V_{1}\left(y_{k} ; I\right) \leq K$ is changed to $V_{4}\left(y_{k} ; I\right) \leq K$ or $V_{2}\left(y_{k} ; I\right) \leq K$. 
In the case where the sequence of functions $\left\{y_{k}\right\}$ is such that $y_{k}: S \rightarrow$ $R, S \subset R^{l}$, we obtain a characterization of those sequences of functions which have pointwise convergent subsequences.

Definition 5.3. Let $S$ be a nonempty subset of $R^{l}$ and $\left\{y_{k}\right\}$ be a sequence of functions, $y_{k}: S \rightarrow R$. We say that the set $\left\{y_{k}\right\}$ is equioscillatory if for each $s \in S$ there exists a nested neighborhood basis of 0 of radii $\epsilon(n, s)$ and for each positive integer $n$ there exist positive integers $H(n)$ and $N(n)$ such that if $k, j \geq H(n)$ and $\left(y_{k}-y_{j}, P\right)$ is a proper pair then $P$ contains no more than $N(n)$ points $x$ for which $\left|y_{k}(x)-y_{j}(x)\right|>\epsilon(n, x)$.

Theorem 5.4. Let $S$ be a nonempty subset of $R^{l}$ and $\left\{y_{k}\right\}$ be a sequence of functions, $y_{k}: S \rightarrow R^{q}$. The sequence $\left\{y_{k}\right\}$ has a subsequence which is pointwise convergent if and only if it has a subsequence $\left\{h_{k}\right\}$ which is pointwise bounded and $\left\{h_{k}^{(i)}\right\}$ is equioscillatory for $i=1,2, \ldots, q$.

Proof. Apply Theorem 5.1 to $\left\{y_{k}\right\}$ coordinatewise to get a convergent subsequence. On the other hand if $\left\{y_{k}\right\}$ has a subsequence $\left\{h_{k}\right\}$ which converges pointwise to $y$, then it must be pointwise bounded. By letting $\epsilon(n, t)$ $=\sup _{k, j \geq n}\left\{\left\|h_{k}(t)-h_{j}(t)\right\|\right\}, N(n)=0$ and $H(N)=n$, then for $k, j \geq H(n)$ we have $\left\|h_{k}(t)-h_{j}(t)\right\|<\epsilon(n, t)$ for all $t$ so that $\left\{h_{k}^{(i)}\right\}$ is an equioscillatory sequence for $i=1,2, \ldots, q$.

The following two notes relate sequences equioscillatory and sequences being Cauchy.

Note 1. The sequence $\left\{y_{k}\right\}, y_{k}: S \rightarrow R, S \subset R^{l}$, is equioscillatory with $N(n)=0$ if and only if $\left\{y_{k}\right\}$ is pointwise Cauchy.

Proof. If $\left\{y_{k}\right\}$ is equioscillatory with $N(n)=0$ then for $\delta(x)>0$ there exists a positive integer $n$ such that $\left|y_{k}(x)-y_{j}(x)\right|<\epsilon(n, x)<\delta(x)$ for all $k, j \geq H(n)$. If $\left\{y_{k}\right\}$ is pointwise Cauchy choose $\epsilon(n, x)=\sup _{k, j \geq n}\left\{\left|y_{k}(x)-y_{j}(x)\right|\right\}$, $N(n)=0$ and $H(n)=n$ so that $\left\{y_{k}\right\}$ is equioscillatory.

Note 2. The sequence $\left\{y_{k}\right\}, y_{k}: S \rightarrow R, S \subset R$, is equioscillatory with $N(n)=0$ and $\epsilon(n, x)=\epsilon_{n}$ a nested neighborhood basis of zero if and only if $\left\{y_{k}\right\}$ is uniformly Cauchy.

Proof. If $\left\{y_{k}\right\}$ is equioscillatory with $N(n)=0$ and the nested neighborhood basis of 0 is $\epsilon(n, x)=\epsilon_{n}$ then for $\delta>0$ there exists a positive integer $n$ such that $\epsilon_{n}<\delta$ and $\left|y_{k}(x)-y_{j}(x)\right|<\epsilon_{n}=\epsilon(n, x)$ for all $k, j \geq H(n)$. Conversely if $\left\{y_{k}\right\}$ is uniformly Cauchy then for the nested neighborhood basis of radii $\epsilon(n, x)=1 / n$ there exists an $H(n)$ such that for $k, j \geq H(n), \mid y_{k}(x)-$ $y_{j}(x) \mid<1 / n=\epsilon(n, x)$ so $\left\{y_{k}\right\}$ is equioscillatory with $N(n)=0$.

\section{REFERENCES}

1. P. Browne, A singular multi-parameter eigenvalue problem in second order ordinary differential equations, J. Differential Equations 12 (1972), 81-94. 
2. P. Hartman, Ordinary differential equations, Wiley, New York, 1964. MR 30 $\# 1270$.

3. L. H. Hildebrandt, Introduction to the theory of integration, Pure and Appl. Math., vol. 13, Academic Press, New York, 1963. MR 27 \#4900.

4. E. W. Hobson, The theory of functions of a real variable and the theory of Fourier's series, Dover, New York, 1927.

5. L. K. Jackson and K. Schrader, Existence and uniqueness of solutions of boundary value problems for third order differential equations, J. Differential Equations 9 (1971), 46-54. MR 42 \#4813.

6. W. A. J. Luxemburg and A. C. Zaanen, Riesz spaces. Vol. 1, North-Holland, Amsterdam, 1971.

7. C. W. McArthur, Convergence of monotone nets in ordered topological vector spaces, Studia Math. 34 (1970), 1-16. MR 41 \#197.

8. E. J. McShane, Integration, Princeton Univ. Press, Princeton, N. J., 1944. MR 6, 43.

9. S. E. Newman, Measure algebras and functions of bounded variation on idempotent semigroups, Bull. Amer. Math. Soc. 75 (1969), 1396-1400. MR 40 \#4778.

10. A. L. Peressini, Ordered topological vector spaces, Harper \& Row, New York, 1967. MR 37 \#3315.

11. F. Ramsey, On a problem of formal logic, Proc. London Math. Soc. (2) 30 (1930), 264-286.

12. The foundations of mathematics, Harcourt-Brace, New York, 1931.

13. K. W. Schrader, A pointwise convergence theorem for sequences of continuous functions, Trans. Amer. Math. Soc. 159 (1971), 155-163. MR 43 \#6621.

14. - A generalization of the Helly selection theorem, Bull. Amer. Math. Soc. 78. (1972), 415-419. MR 45 \#8788.

15. K. Schrader and J. Thornburg, Sufficient conditions for the existence of convergent subsequences, Pacific J. Math. (to appear).

16. H. H. Schaefer, Topological vector spaces, Macmillan, New York, 1966. MR $33 \# 1689$.

DE PARTMENT OF MATHEMATICS, UNIVERSITY OF MISSOURI, COLUMBIA, MISSOURI 65201

Current address: Burroughs Corporation, 1113 North Broadway, Lexington, Kentucky 40505 